\title{
Lapatinib Regimen
}

National Cancer Institute

\section{Source}

National Cancer Institute. Lapatinib Regimen. NCI Thesaurus. Code C160073.

A chemotherapy regimen consisting of lapatinib that may be used in the treatment of bone cancer. 